Voix et Images

\title{
À la croisée des champs artistique et littéraire : le livre d'artiste au Québec, 1900-1980
}

\section{Silvie Bernier}

Volume 11, numéro 3 (33), printemps 1986

Yolande Villemaire

URI : https://id.erudit.org/iderudit/200586ar

DOI : https://doi.org/10.7202/200586ar

Aller au sommaire du numéro

Éditeur(s)

Université du Québec à Montréal

ISSN

0318-9201 (imprimé)

1705-933X (numérique)

Découvrir la revue

Citer cet article

Bernier, S. (1986). À la croisée des champs artistique et littéraire : le livre

d'artiste au Québec, 1900-1980. Voix et Images, 11(3), 528-536.

https://doi.org/10.7202/200586ar d'utilisation que vous pouvez consulter en ligne.

https://apropos.erudit.org/fr/usagers/politique-dutilisation/ 


\title{
Ā la croisée des champs artistique et littéraire: le livre d'artiste au Québec, 1900-1980
}

\author{
par Silvie Bernier, Université de Sherbrooke
}

Aux confins du livre illustré et du livre-objet, le livre d'artiste occupe un champ difficilement délimitable et reçoit des divers agents du milieu artistique des définitions différentes. Au Québec, l'une des premières à tenter une définition rigoureuse du livre d'artiste a été Claudette Hould, professeure d'histoire de l'art à l'UQAM et responsable du Répertoire des livres d'artistes au Québec, 1900-1980' dans lequel elle énonce les critères qui lui ont servi de base pour sa sélection. On peut les ramener à quatre. Tout d'abord, le livre d'artiste doit être constitué à la fois d'un texte et d'une ou plusieurs illustrations. Ces illustrations sont nécessairement originales, c'est-à-dire qu'elles excluent tout procédé de reproduction photomécanique. Claudette Hould élimine également les œuvres qui ont avant tout une vocation plastique, en d'autres termes les livres-objets, mais, d'autre part, elle rejette aussi bien les livres dans lesquels l'illustration ne possède aucune autonomie vis-à-vis du texte et dont l'unique fonction est de le "doubler»: elle range plutôt ce type d'ouvrages dans la catégorie des livres illustrés. À ces critères, elle en ajoute d'autres plus facultatifs, tels l'édition à tirage limité, le papier de qualité et les exemplaires signés par l'auteur et par l'artiste.

Mais malgré le souci de rigueur de Claudette Hould, ses critères demeurent sujets à interprétation. Ainsi la notion d'estampe originale fait-elle l'objet d'opinions divergentes. Premièrement, elle limite grandement la production de livres illustrés par des artistes en excluant grand nombre de ceux dont les illustrations sont reproduites de façon mécanique et qui supposent pourtant une collaboration entre artistes et écrivains. Ensuite, si certains acceptent la gravure d'interprétation, c'est-à-dire celle réalisée par un artisan à partir de l'œuvre originale d'un artiste ${ }^{2}$, d'autres n'admettent que l'ouvrage accompli intégralement par un seul artiste. Il faut préciser toutefois que l'auteure aligne sa définition sur celle établie par le Conseil de la gravure du Québec dans son Code d'éthique de l'estampe originale ${ }^{3}$. Quant à l'exigence d'autonomie de l'image par rapport au texte, elle demeure vague et fait intervenir inévitablement un jugement de valeur. En effet, à quel moment peut-on dire d'une image qu'elle est originale et autonome? Claudette Hould elle-même admet déroger à ses principes en intégrant à son corpus Courriers des villages (illustré par Rodolphe Duguay), conservé pour la qualité de ses bois gravés, même si les images de l'artiste servent avant tout d'illustrations au texte. Le même problème se pose pour des ouvrages tels que Vieilles choses, vieilles gens de Georges Bouchard ou Metropolitan Museum de Ro- 
bert Choquette, illustrés tous deux par Edwin Holgate. Pour ce qui est de l'exclusion des livres-objets, si le principe semble évident, son application l'est beaucoup moins et il devient parfois difficile d'établir, dans le cas de certaines ouvres limites, si ce qui l'emporte est leur aspect matériel ou leur contenu littéraire. D'autant plus qu'un examen des œuvres énumérées au répertoire nous montre que très souvent le texte n'est qu'un prétexte à l'élaboration d'une ou de plusieurs cuvres plastiques. C'est le cas entre autres de Graff Dinner aux éditions Graff, mais également de bon nombre d'ouvrages dans lesquels le texte devient nettement secondaire par rapport à son illustration.

Dans un numéro spécial d'Études françaises portant sur «l'objet-livre», Jean-Marcel Duciaume ${ }^{4}$ reprend la définition de Claudette Hould, la critique et propose ses propres critères qui s'énoncent comme suit: production artisanale (en dehors des circuits de l'édition commerciale), papiers faits main, typographie soignée, caractères d'imprimerie choisis pour leur lisibilité et leur beauté, gravures originales, tirage limité, plaques rayées après le tirage. Ces critères, malgré leur précision, demeurent selon nous des critères d'excellence et ne permettent pas d'établir une définition rigoureuse du livre d'artiste. Il faut admettre cependant que c'est en ces mêmes termes que Roland Giguère, l'un des pionniers du livre d'artiste au Québec, conçoit le travail éditorial fait chez Ertas.

Finalement, la plus récente définition est donnée par les responsables du Concours national du livre d'artiste qui avait lieu en automne 1983 et qui était organisé par la Galerie Aubes de Montréal. Ils délimitent trois types de productions admises au concours:

[...]1) le livre illustré à tirage limité comportant un texte et une illustration reproduite de façon non mécanique; 2) le livre d'artiste à grand tirage; toute forme de reproduction est acceptable. Ce livre doit être réalisé par un ou des artistes uniquement; 3) le livre-objet: livre tiré à un seul exemplaire réalisé par un ou des artistes $^{6}$.

Ainsi, on élargit la définition de Claudette Hould en ajoutant à la liste des ouvrages constitués non seulement de gravures originales mais aussi de toute autre forme de reproduction (pour autant que ces livres sont conçus par un artiste et non par un éditeur). On intègre également les livres-objets. Cette typologie a, bien sûr, l'avantage de tenir compte de la production actuelle des artistes québécois dans laquelle le livre-objet est prédominant. Par contre, elle n'accorde au texte qu'une importance facultative puisque plusieurs livresobjets ne maintiennent avec le livre qu'une ressemblance formelle. Elle offre également une ouverture du côté des livres illustrés de reproductions non originales.

Quant à nous, c'est à la définition de Claudette Hould que nous nous sommes ralliée. Nous sommes consciente de ses limites mais elle demeure, selon nous, la plus fonctionnelle ${ }^{7}$. D'une part, elle a l'avantage de tenir compte d'un corpus sur lequel il est possible de travailler et d'autre part, 
malgré l'imprécision de ses limites, elle cherche à circonscrire un certain type de production qui tient compte à la fois du texte et de l'illustration. Ce corpus, allant de 1900 à 1980 , permet de saisir le livre d'artiste dans son historicité et son évolution. C'est ainsi qu'il apparaîtra comme un phénomène daté qui naît véritablement dans les années quarante pour disparaître peu à peu aujourd'hui, remplacé dans sa formule par le livre-objet. C'est un peu cette histoire que nous nous proposons de faire ici en reprenant les diverses étapes de l'évolution du livre d'artiste au Québec, en insistant surtout sur les institutions qui l'ont rendu possible.

On peut, en fait, articuler les étapes de la production du livre d'artiste autour d'une seule maison d'édition: avant, pendant et après Erta. Durant les années vingt et trente de même qu'au début des années quarante, Claudette Hould ne dénombre que sept livres d'artistes: Vieilles choses, Vieilles gens (1928) édité chez Louis Carrier, le Grand silence blanc (1928) de L.-F. Rouquette illustré par Clarence Gagnon et édité à Paris chez Mornay, le Cantique des cantiques de Salomon (1931), avec des illustrations de Cécile Buller et paru aux éditions du Raisin à Paris, Poèmes (1934) d'Alain Grandbois et d'un illustrateur anonyme édité en Chine par Yan Hung Tin Wu Chii, Vitrail (1939) rédigé et illustré par Cécile Chabot, imprimé sous l'étiquette de la maison Bernard Valiquette mais aux frais de l'auteure et finalement Courriers des villages (1940) paru aux éditions du Bien public. Ces livres sont des livres illustrés mais leur présentation particulièrement soignée et les tirages limités les rapprochent du livre d'artiste. Ce qui frappe également dans ce répertoire, c'est l'importance des publications réalisées hors du Québec, ce qui ramène les productions québécoises au nombre de quatre. Tous ces ouvrages sont le fait d'éditeurs différents ou alors de comptes d'auteur. En somme, il n'existe pas à cette époque au Québec de véritable politique éditoriale favorisant le livre d'artiste.

En 1949, paraît le recueil Faire naître de Roland Giguère et d'Albert Dumouchel. Ce titre annonciateur signale l'avènement d'une nouvelle maison d'édition ayant pour vocation de publier des livres d'artistes ${ }^{8}$. Au cours de la décennie cinquante, cette maison publie dix-sept livres d'artistes ${ }^{9}$, ce qui représente un nombre important pour un seul éditeur. La fondation de Erta est directement liée à l'école des Arts graphiques où Roland Giguère étudiait. C'est d'ailleurs sur les presses de l'école que Giguère imprima ses premiers livres. Comme le souligne Claudette Hould: [...]l'histoire du livre d'artiste colle donc de très près à celle de la gravure moderne québécoise dont les débuts dans les années cinquante correspondent aux recherches d'Albert Dumouchel et de ses premiers élèves à l'Institut [ sic] des Arts graphiques ${ }^{10}$. C'est donc dès le départ une institution du champ artistique qui est à la base du développement du livre d'artiste. Il faut dire cependant que Roland Giguère, malgré sa formation de typographe, est également poète. Il réunit donc toutes les qualités nécessaires pour promouvoir le livre d'artiste puisqu'il appartient à la fois au monde des arts et à celui des lettres. Mais Erta est avant tout une maison d'édition de poésie où le texte demeure prioritaire. Même si les images qui accompagnent le texte ne sont pas à proprement 
parler des illustrations, elles s'en inspirent d'une façon ou d'une autre. Selon Roland Giguère, les livres publiés par Erta durant les années cinquante n'étaient pas de véritables livres d'artistes. Là-dessus, il rejoint la position de Jean-Marcel Duciaume qui prétend que ce type d'ouvrage doit afficher un souci de présentation évident (papier grand format, typographie faite à la main, etc.) ce qui n'était pas le fait des ouvrages imprimés durant les années cinquante. Giguère les qualifie de [...] petites plaquettes, plus ou moins bien faites avec des bouts de ficelle, avec rien du tout ". Ils correspondent néanmoins aux critères de Claudette Hould, d'autant plus qu'existe chez Erta une volonté réelle de susciter une collaboration étroite entre artistes et écrivains.

À la fin des années cinquante naît la maison d'édition Goglin qui doit elle aussi sa création à l'initiative d'anciens élèves de l'école des Arts graphiques: Françoise Bujold, Richard Lacroix, Janine Leroux. En 1958, paraît le premier ouvrage, la Fille unique, constitué de poèmes et de bois gravés de Françoise Bujold. L'année suivante, la maison édite quatre livres d'artistes qui se démarquent par leur nouveauté, entre autres celui de Françoise Bujold, l'Ile endormie, illustré par les enfants du Centre d'Art de Percé ainsi que Sept eaux-fortes, constitué d'une série de gravures de Françoise Bujold, Richard Lacroix, Janine Leroux et Marie-Anastasie (préface de Guy Robert). Cette maison, qui n'a vécu que deux ans et qui a regroupé un nombre très restreint d'artistes et d'écrivains, a lancé néanmoins l'édition du livre d'artiste de luxe en misant fortement sur l'aspect matériel des ouvrages publiés. Les livres sont dorénavant de grand format et respectent les normes de qualité qu'on attend d'un tel type d'ouvrages. On remarque également l'importance nouvelle accordée à l'image, particulièrement dans Sept eaux-fortes où le texte n'est constitué que d'une préface.

Les années soixante sont moins marquantes en ce qui concerne le livre d'artiste. La production globale passe de vingt-quatre titres pour les années cinquante à trente-deux. On ne signale pas non plus d'éditeurs dominants, mis à part peut-être les éditions du Songe et les éditions Sentinelle qui publient chacun trois livres. C'est finalement le compte d'auteur qui regroupe le plus d'ouvrages même si, encore là, ils se limitent à cinq. Il faut mentionner l'apparition de quelques nouvelles maisons dans le champ éditorial du livre d'artiste. Entre autres, Sentinelle qui publie exclusivement des œuvres de Françoise Bujold, illustrées de dessins d'enfants. Cette maison reprend, en quelque sorte, le type de production de Goglin. Les éditions du Songe sont, ni plus ni moins, la maison de Guy Robert. Il y publie en 1969 trois ouvrages, Intrême-Orient, Syntaxe pour Lardera, et Trans-apparence. Le premier est illustré par Monique Charbonneau et les deux derniers par Berto Lardera. Par la suite, dans les années soixante-dix, il accroît la production de la maison de quatre nouveaux livres dont trois sont de sa plume, Jordi Bonet (1977) accompagné de deux cuvres de l'artiste et $\mathbf{3 5 0}$ millions d'années lumière illustré également par Stelio Sole. Finalement, en 1980, Guy Robert publie Suite québécoise Charlevoix qui présente sept œuvres d'Albert Rous- 
seau. Les éditions du Songe sont donc, comme on peut le constater, nettement orientées vers le champ artistique et le but des publications est très souvent de faire connaitre un artiste au public (Syntaxe pour Lardera, Stelio Sole, Jordi Bonet). De plus, la part accordée à l'illustration est considérable et souvent plus importante que le texte.

D'autres maisons sont à signaler pour la période des années soixante. Citons les éditions de l'Estérel qui publient deux textes de Michel Beaulieu accompagnés d'estampes originales de Roland Pichet; les éditions La Frégate qui débutent en publiant des monographies d'artistes: Suzor-Côté (1967) et plus tard Maurice Cullen (1978), René Richard (1974), Henri Masson (1976) et poursuivent avec des réimpressions de classiques, Flammes de l'amour ${ }^{12}$ (1973), la Montagne secrète (1975), Agaguk (1971), Menaud maître-draveur (1979). La maison Fides réédite elle aussi des œuvres connues du corpus québécois: Poésies d'Émile Nelligan (1967) ${ }^{13}$, Poèmes d'Alain Grandbois (1970), le Survenant (1977), Maria Chapdelaine (1980). Mentionnons également l'unique publication des éditions Déom dans la série du livre d'artiste, parue dans la collection "Poésie canadienne» dirigée par Guy Robert : Miroir de lumière (1965), entièrement réalisée par Marie-Anasthasie. Et finalement LE ÇA IRA ( 1967) de José Pierre illustré par Mimi Parent aux éphémères éditions Surréalistes, ainsi que Énigmes (1964) de Harold Town paru chez McClelland and Stewart à Toronto.

Ce qu'on retient surtout de la décennie soixante, c'est le nouveau rôle d'éditeur assumé par des institutions proprement artistiques telles que des galeries (Maeght, Agnès Lefort, Godard Lefort), des écoles (ateliers de l'École des Beaux-Arts de Montréal), ou des ateliers de gravure (la Guilde graphique). Cette tendance se poursuivra et s'accentuera dans les années soixante-dix avec la mise en place de tout un réseau institutionnel relié spécifiquement à l'art de la gravure.

En 1968, la loi du dépôt légal au gouvernement du Québec rend obligatoire le dépôt à la Bibliothèque nationale de tout ouvrage publié (la loi du dépôt légal à Ottawa date de 1933). La bibliothèque se doit, en contrepartie, d'acheter les livres évalués à plus de trois cents dollars. Le livre d'artiste prend donc de l'intérêt puisqu'il permet à l'auteur d'avoir dès le départ deux acheteurs assurés (les deux bibliothèques nationales). Au cours de la décennie soixante-dix, cent quatre-vingt treize livres d'artistes sont publiés, ce qui représente un accroissement considérable par rapport aux années précédentes. Cette fois, certains éditeurs se démarquent aisément. En tête, les ateliers de la Guilde Graphique qui publient vingt ouvrages, les ateliers Graff, dix-huit, Michel Nantel, treize, La Frégate, huit, Art global, cinq, la Minerve, quatre, sans calculer les cinquante comptes d'auteur qui constituent plus du quart de la production totale. Ces comptes d'auteur sont significatifs pour leur importance numérique mais aussi parce qu'ils sont majoritairement assumés par l'artiste plutôt que par l'écrivain. Contrairement à Metropolitan Museum qui était co-édité par Robert Choquette et Edwin Holgate, les ouvrages plus récents se font aux frais de l'artisan-graveur uniquement. Ceci démontre, 
encore une fois, que le livre d'artiste concrétise une initiative du milieu artistique bien plus que du monde des lettres.

On notera l'importance des éditeurs qui ont comme origine des ateliers de gravure (à la Guilde Graphique s'ajoutent l'atelier de réalisations graphiques, les ateliers Graff et le centre de conception graphique), des galeries (Gilles Gheerbrant, Gilles Corbeil, Maeght, Mira Goddart, Parallèles) ou qui présentent d'une façon ou d'une autre des liens avec le champ artistique (Art global, Collection du livre d'art de Loto-Québec, Famille des arts de l'Université du Québec à Montréal, etc.). Par les ouvrages qu'elles publient, ces maisons confirment leurs attaches au secteur des arts. Prenons l'exemple des ateliers de la Guilde Graphique qui lancent leurs activités d'édition en 1969. Leurs livres d'artistes sont constitués majoritairement de planches accompagnées de courts textes. De toute évidence ces publications mettent l'accent sur les gravures. L'écrit semble tout juste utile à justifier l'appellation de «livre». On peut en dire autant des éditions Graff dont les publications sont souvent le fait d'artistes qui s'adonnent, pour l'occasion, à l'écriture. Un autre type de production est celui des éditions Art global fondées en 1975, qui visent à promouvoir des artistes par le biais de grands textes littéraires tels que Prochain épisode, Salut Galarneau!, La Guerre, yes sir!, Kamouraska. Cette fois l'écrit a de l'importance quantitativement mais présente peu d'intérêt puisqu'il est généralement connu du lecteur. Les éditions Michel Nantel reprennent tantôt l'une, tantôt l'autre de ces deux politiques. Ils publient donc aussi bien des textes de Robert Choquette ou d'Alfred DesRochers ${ }^{14}$ que des œuvres d'artistes peu connus. Quant aux éditions de la Minerve, elles servent en fait à diffuser la production artistique d'Irénée Lemieux qui s'associe à différents écrivains dans l'élaboration de ses livres d'artistes.

Ce rapide parcours de l'édition montre clairement de quelle façon ce type d'ouvrages quitte peu à peu le champ littéraire pour devenir une production relevant le plus souvent d'institutions artistiques. La prise en charge du livre d'artiste par le champ artistique se reflète dans le contenu des ouvrages où le texte perd de l'importance au profit de l'wuvre plastique. Prenons l'exemple de Metropolitan museum paru en 1939: sur les vingt-neuf pages de l'album, douze sont réservées partiellement à une illustration (en plus de la maquette couverture). Ainsi, environ le sixième de l'espace est consacré à l'œuvre de Holgate. Ces illustrations sont toutes figuratives, quoiqu'elles présentent une certaine stylisation et maintiennent un lien étroit avec le contenu du texte. La situation est bien différente lorsqu'on arrive à la production de la fin des années soixante, avec des maisons comme Graff et le Grainier qui privilégient nettement l'image. C'est le cas d'un ouvrage comme le Grainier d'Yves Préfontaine, publié à la maison du même nom, qui comprend deux pages de textes suivies de sept eaux-fortes de Marie-Anastasie, fondatrice et propriétaire des éditions du Grainier. Les gravures se rapprochent de l'abstraction pure et n'ont qu'un rapport très indirect avec le contenu écrit. En fait, il apparaît assez nettement que le Grainier sert avant tout la production artistique de sa propriétaire. On pourrait citer plusieurs autres exemples de publications récentes où le texte n'est plus qu'un prétexte à la diffusion d'ouvres graphiques. 
Comme nous l'avons vu, cette réduction du rôle de l'écrit dans le livre d'artiste ne se calcule pas nécessairement en nombre de pages mais aussi par le choix des textes édités, qui sont souvent des rééditions d'œuvres célèbres. C'est la politique de maisons d'édition comme Art global, La Frégate, Fides et quelques autres. L'ouvre littéraire et le renom du romancier servent de crédit à l'artiste et lui permettent de populariser ses œuvres. Parfois, le texte en lui-même est inédit mais l'auteur est prestigieux et suffit à susciter l'intérêt d'un public potentiel. Il est intéressant de noter à ce sujet quels écrivains sont le plus souvent mis à contribution. On trouve, entre autres, Émile Nelligan auquel sont consacrés cinq livres d'artistes, Félix-Antoine Savard (quatre), Alfred DesRochers, Rina Lasnier, Félix Leclerc (trois) et Gaston Miron, Fernand Ouellette et Gabrielle Roy (deux). Ces écrivains sont tous connus et n'ont plus besoin de consécration. En fait, le livre d'artiste ne vient que confirmer leur prestige.

Nous avons touché également à une autre formule du livre d'artiste qui est constitué d'un texte et d'une ouvre plastique provenant du même auteur. Il s'agit généralement d'un artiste-graveur qui décide d'accompagner des images d'un écrit souvent poétique. Ici aussi, l'estampe l'emporte sur le contenu littéraire et sert de motivation à la création de l'ouvrage. Citons les titres de Fernand Bergeron, Dans cracrais (1973), Hotel-Motel Beauséjour : bar salon, piscine à l'eau salée, M. Bouchard, prop. (1976), Pour les nuits blanches de Nini de Saint-H la petite (1973) et Tour l'oignon (1969) ou encore Jordi Bonet, Être conscient (1975), Thérèse Boudreau-Cholette, Mutances (1980) et les ouvres de Jean Brodeur, Carl Daoust, Lyne Ouvrard ainsi que plusieurs autres.

Le marché du livre d'artiste est lui aussi révélateur de son orientation artistique. Ce marché est, bien sûr, limité puisque les tirages montent rarement à plus de 500 exemplaires et parfois, atteignent à peine 10 exemplaires. Selon les théories de Pierre Bourdieu ${ }^{15}$, ce type de production se situerait à l'extrême limite dans le champ de la production restreinte. Les acheteurs de livres d'artistes constituent un public amateur qui s'intéresse avant tout aux qualités plastiques des œuvres. Ce sont donc, en plus des deux bibliothèques nationales, des collectionneurs d'œuvre d'art. Ce sont également beaucoup plus les galeries que les libraires qui vendent ou font la promotion des livres d'artistes. Les éditeurs fonctionnent également par souscription, c'est-à-dire qu'ils envoient le projet de livre à un certain nombre d'acheteurs potentiels qui s'engagent ou non à acheter l'ouvrage une fois ce dernier publié. De cette façon, la maison d'édition ou l'artiste sont assurés d'un montant minimum qui leur permet d'assumer les frais encourus par la publication de l'ouvrage.

Ainsi, le livre d'artiste est un moyen pour les artisans-graveurs de proposer un type différent de production qui a en plus l'avantage d'être achetée par les bibliothèques nationales, ce qui n'est pas le cas des simples gravures. Étant donné que les estampes sont vendues en bloc, elles reviennent moins cher l'unité et suscitent l'intérêt des collectionneurs qui peuvent par la suite les revendre au prix fort. Certains éditeurs cependant se refusent à cette 
pratique et font signer aux artistes l'ensemble de l'ouvrage et non chaque gravure séparément.

Si nous avons affirmé précédemment que le livre d'artiste tendait à disparaître peu à peu, c'est en fait que la production récente montre que la formule qui consiste à joindre le texte d'un auteur à l'œuvre d'un artiste semble être devenue un peu désuète ou du moins constitue un modèle plutôt traditionnel. On remarque que les nouvelles propositions avant-gardistes tendent plutôt vers le livre-objet comme le prouve le Concours national du livre d'artiste où deux prix sur trois ont été attribués à des livres-objets de façon à rendre compte plus adéquatement du type d'œuvres soumises au jury.

En définitive, la formule du livre d'artiste qui consiste à créer une unité entre l'œuvre d'un écrivain et celle d'un artiste semble avoir été à l'avantage de ce dernier. Le marché de cette forme de production s'est rapidement déplacé du milieu littéraire vers le milieu artistique. Le passage de la loi du dépôt légal du Québec qui stipule que la Bibliothèque nationale doit acheter tout livre ayant une valeur supérieure à $300 \$$ a encouragé la production de livres coûteux qui, pour justifier leur prix, se doivent de miser avant tout sur l'aspect matériel de l'œuvre ce qui finit par éclipser inévitablement le texte.

Les livres-objets vont encore plus loin et éliminent parfois complètement l'écrit. Dans ces cas-limites, le livre n'est plus qu'un thème d'inspiration et le rapprochement avec l'œuvre plastique demeure lointain. Mentionnons, comme exemples, un bottin téléphonique entièrement collé et sculpté où les caractères d'imprimerie sont rendus indéchiffrables; ou encore un bloc de métal rectangulaire imitant la forme d'un livre fermé ${ }^{16}$. Ainsi, le livre d'artiste s'oriente de plus en plus vers les arts plastiques, à mi-chemin entre la sculpture et le «ready-made», et trouve désormais sa place dans les galeries d'art bien plus que dans les librairies.

1. Claudette Hould, Répertoire des livres d'artistes au Québec, 1900-1980, avec la collaboration de Sylvie Laramée, Montréal, ministère des Affaires culturelles, 1982, $240 \mathrm{p}$.

2. Citons en exemple Prochain épisode d'Hubert Aquin illustré par Louis et Violette Desaulniers ainsi que Yannick Ballif d'après des œuvres de Fernand Toupin. Le livre est signé par Fernand Toupin.

3. Code d'éthique de l'estampe originale, Conseil de la gravure du Québec, 1982, $47 \mathrm{p}$.

4. Jean-Marcel Duciaume, "Le livre d'artiste au Québec: contribution à une histoire», Études françaises, vol. 18, no 2, p. 89-98.

5. Entrevue réalisée par Richard Giguère et Hélène Lafrance avec Roland Giguère à l'Atelier Giguère-Tremblay ( 3684 boul. Saint-Laurent), novembre 1983.

6. Règlements du Concours national du livre d'artiste, Galerie Aubes, Montréal, 1983.

7. Voir l'article de Richard Giguère, "Les éditions Erta: un surréalisme sans frontières", dans l'Édition littéraire au Québec de 1940 à 1960, coll. "Cahiers d'études littéraires et culturelles», no 9, Université de Sherbrooke, 1985, p. 139-177.

8. Il n'existe pas à notre connaissance de répertoire de tous les livres illustrés au Québec.

9. Roland Giguère dans une entrevuc avec Richard Giguère et Hélène Lafrance nie avoir fait du livre d'artiste durant les années cinquante. les reproductions étant, dit-il, reproduites de façon photo-mécanique. Il mentionne toutefois qu'il faisait en plus du 


\section{VOIX \& IMAGES/33, printemps 1986}

tirage habituel un tirage à part de 10 à 15 exemplaires et qui, celui-là, était constitué de gravures originales. Il est donc tout à fait légitime, selon la définition de Claudette Hould, de considérer ces publications comme des livres d'artistes.

10. C. Hould, op. cit., p. 125.

11. Voir entrevue citée avec Roland Giguère.

12. Flammes de l'amour est un recueil de poèmes de Burns, Carew, Cordeleau, Coleridge, Cotin, Cotton, Cunningham, De la Fresnaye, De Maleville, Dyer, Forneret, Herrick, Hugo, Johnson. Lodge, Lovelace, Malherbe, Maynard, Musset, Parniyk Poe, Ronsard, Sarsin, Shakespeare, Spencer, Wordsworth, Waller avec des sérigraphies de Hugues de Jouvancourt.

13. Cet ouvrage est édité en collaboration avec la galerie Gilles Corbeil.

14. Suite marine (1976) de Robert Choquette illustré par Indira Nair et Élégies pour l'épouse en-allée (1973) d'Alfred DesRochers illustré par Roland Pichet.

15. Pierre Bourdieu, "Le marché des biens symboliques", l'Année sociologique, no 22 , février 1971, p. 49-126.

16. Ces ceuvres sont tirées de l'exposition de livres d'artistes ayant eu lieu à la Galerie Aubes à la suite du Concours national de livres d'artistes à l'automne de 1983.

\section{BIBLIOGRAPHIE}

BOURDIEU, Pierre. "Le marché des biens symboliques", l'Année sociologique, no 22. février 1971, p. 49-126.

DAIGNEAULT, Gilles, "L'Aventure d'Art global», Vie des Arts, no 46, printemps 1967. p. 25-27.

DUCIAUME. Jean-Marcel. «Le livre d'artiste au Québec: contribution à une histoire». Êtudes françaises, vol. 18, no 2, automne 1982, p. 89-98.

GAGNON, Claude-Lyse. «La Guilde Graphique», Vie des Arts, no 46, printemps 1967, p. 61-62.

GIGUĖRE, Richard et LAFRANCE, Hélène. Entrevue réalisée avec Roland Giguère à l'Atelier Giguère-Tremblay ( 3684 boul. Saint-Laurent) en novembre 1983.

GIGUERE, Richard. "Les éditions Erta: un surréalisme sans frontières», dans l'Édition littéraire au Québec de 1940 à 1960, coll. "Cahiers d'études littéraires et culturelles" no 9. Université de Sherbrooke, 1985, p. 139 à 177.

GIGUËRE, Roland. "Une aventure en typographie: des Arts graphiques aux éditions Erta", Etudes françaises. vol. 18, no 2, automne 1982, p. 89-104.

GIGUERE, Roland. "Aux ateliers de lithographie Desjobert". Vie des Arts, no 17, Noël 1959, p.40-46.

GILLON, Michèle. "Les Graffofones de Graff", Vie des Arts, no 71, été 1973, p. 23-28.

HÉNAULT, Gilles. "Au début des années cinquante: Erta-Arts Graphiques et Poésie", Vie des Arts, no 90, printemps 1978, p. 23-28.

HOULD, Claudette. Répertoire des livres d'artistes au Québec, 1900-1980, avec la collaboration de Sylvie Laramée. Montréal, ministère des Affaires culturelles, 1982, $240 \mathrm{p}$.

PARADIS, André. “Roland Giguère: l'éditeur», Vie des Arts, no 9, Noël 1957, p. 29.

ROUSSAN; Jacques de. "Michel Nantel, artisan du livre», Vie des Arts, no 83, été 1976, p. 59-61.

TOURANGEAU, Jean. «L'ARG», Vie des Arts, no 90, printemps 1978, p. 41-45. 